\title{
Low-Cost Open-Source Voltage and Current Monitor for Gas Metal Arc Weld 3D Printing
}

\author{
A. Pinar, ${ }^{1}$ B. Wijnen, ${ }^{2}$ G. C. Anzalone, ${ }^{2}$ T. C. Havens, ${ }^{1,3}$ P. G. Sanders, ${ }^{2}$ and J. M. Pearce ${ }^{1,2}$ \\ ${ }^{1}$ Department of Electrical \& Computer Engineering, Michigan Technological University, Houghton, MI 49931, USA \\ ${ }^{2}$ Department of Materials Science \& Engineering, Michigan Technological University, Houghton, MI 49931, USA \\ ${ }^{3}$ Department of Computer Science, Michigan Technological University, Houghton, MI 49931, USA
}

Correspondence should be addressed to J. M. Pearce; pearce@mtu.edu

Received 24 March 2015; Accepted 22 June 2015

Academic Editor: Christos Riziotis

Copyright (c) 2015 A. Pinar et al. This is an open access article distributed under the Creative Commons Attribution License, which permits unrestricted use, distribution, and reproduction in any medium, provided the original work is properly cited.

\begin{abstract}
Arduino open-source microcontrollers are well known in sensor applications for scientific equipment and for controlling RepRap 3D printers. Recently low-cost open-source gas metal arc weld (GMAW) RepRap 3D printers have been developed. The entry-level welders used have minimal controls and therefore lack any real-time measurement of welder voltage or current. The preliminary work on process optimization of GMAW 3D printers requires a low-cost sensor and data logger system to measure welder current and voltage. This paper reports on the development of a low-cost open-source power measurement sensor system based on Arduino architecture. The sensor system was designed, built, and tested with two entry-level MIG welders. The full bill of materials and open source designs are provided. Voltage and current were measured while making stepwise adjustments to the manual voltage setting on the welder. Three conditions were tested while welding with steel and aluminum wire on steel substrates to assess the role of electrode material, shield gas, and welding velocity. The results showed that the open source sensor circuit performed as designed and could be constructed for $<\$ 100$ in components representing a significant potential value through lateral scaling and replication in the $3 \mathrm{D}$ printing community.
\end{abstract}

\section{Introduction}

The development of low-cost self-replicating rapid prototypers has evolved from early $3 \mathrm{D}$ printers that could fabricate approximately half of their components from polymers to tools now capable of printing in a wide variety of materials [1-4]. Of widespread industrial interest is a low-cost metal 3D printer capable of printing both steel [5] and aluminum parts [6]. These open-source metal 3D printers can be fabricated for less than \$1,200 [5] using a conventional metal inert gas (MIG) welder and controlled with open-source electronic boards (normally Arduino compatible); they reduce the costs of metal 3D printing by two orders of magnitude and make the technology far more accessible for small and medium sized enterprises. In order to minimize costs, the consumergrade MIG welders used for RepRap 3D metal printing contain minimal controls and therefore lack any real-time measurement of welder voltage or current. The preliminary work on process optimization of these printers requires a lowcost sensor and data logger system to measure current and voltage of the gas metal arc welders (GMAW).

Arduino microcontrollers are already established for use in low-cost high-quality scientific and engineering equipment [7-12]. For example, they have been used for oceanographic research [13], mass spectrometry [14,15], microscopy [16], vision research [17], Skinner boxes [18], mechatronics [19], optics [10, 20], electrophoresis [21], and imaging [22, 23]. Most importantly for this sensor application, Arduinos have applied to systems for sensors for open hardware [24] including colorimetry [25], nephelometry [26], pressure monitoring [27], smart plugs [28], and in vivo optimal imaging [29]. In addition, Arduinos have been demonstrated for field sensing operations like soil moisture [30], developing a wireless sensor node to monitor poultry farms [31] and an ambient sensor cloud system using OpenFS (open field server) for high-throughput phenotyping [32]. Arduinos are 


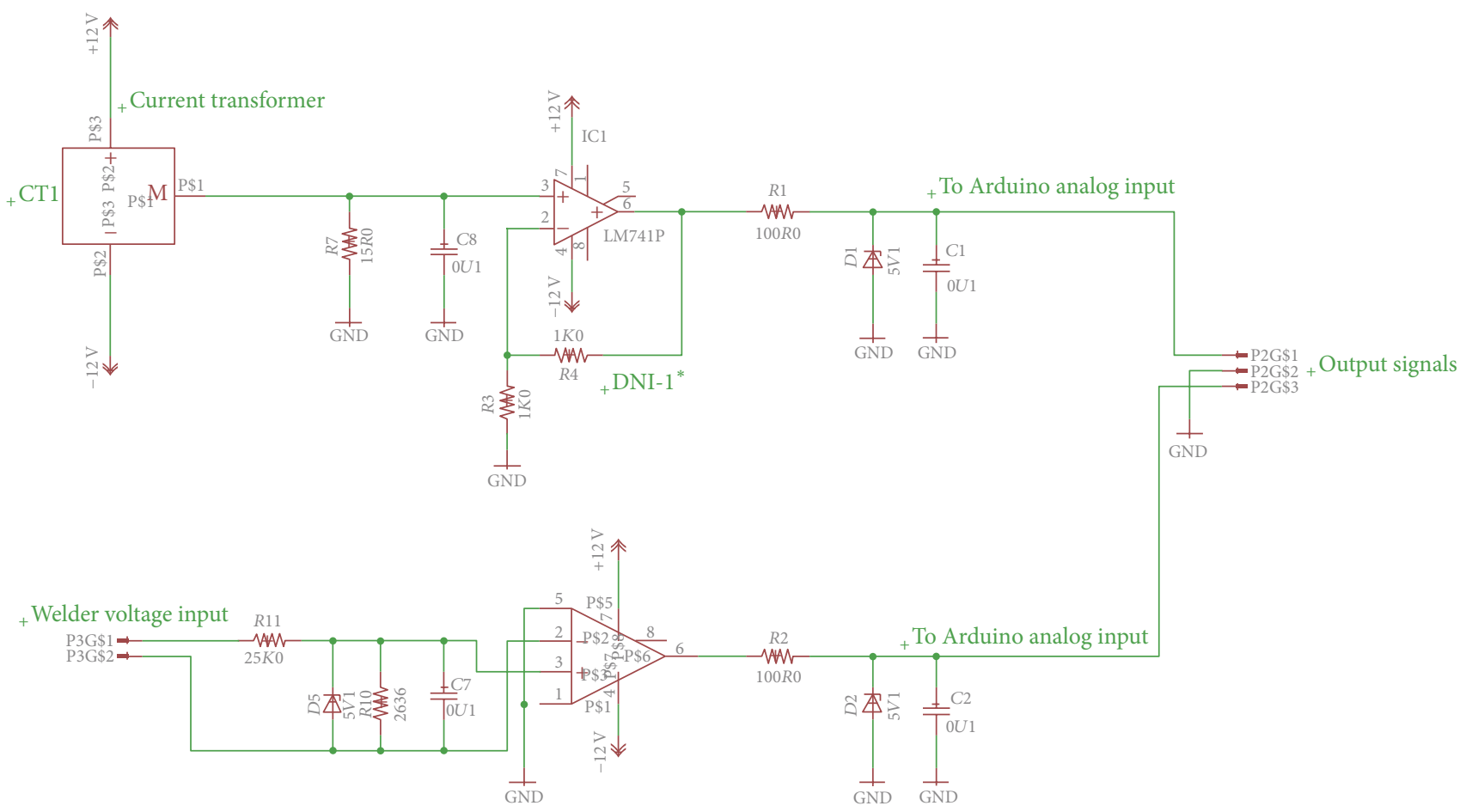

${ }_{+}$DNI- ${ }^{*}$ : replace with 0 ohm jumper for unity gain

${ }_{+} \mathrm{R} 4$ can be changed for nonunity gain

FIGURE 1: Current and voltage sensing circuitry.

also an established technology for controls [33], used as controllers on numerous challenging projects such as for aerial vehicles [34], robots [35, 36], and microfluidics [37].

As this track record has been established and Arduino microcontrollers are already used for controlling the $3 \mathrm{D}$ printer, this paper reports on the development of a low-cost open-source power measurement sensor system based on the Arduino architecture. The current/voltage sensing system was designed and built and used to collect measurements of welder voltage and current in real-time while welding beads with the RepRap 3D printers. The full bill of materials and electrical schematics are provided. The system was tested on two entry-level MIG welders: a Millermatic 140 and a Millermatic 190, both from Miller Electric. Voltage and current were measured while making stepwise adjustments to the manual voltage setting on the welder. Three conditions were tested while welding with steel and aluminum wire on steel substrates to assess the role of electrode material, shield gas, and welding velocity. The results are discussed and future work is outlined to improve low-cost GMAW 3D printing using the open-source sensor system.

\section{Methods and Materials}

2.1. Design. The electric schematics for the current-voltage sensor measurement system are shown in Figure 1 and the circuits for input power protection, indication, and conditioning are shown in Figure 2. The complete bill of materials is shown in Appendix A (in Supplementary Material available online at http://dx.doi.org/10.1155/2015/876714). As discussed below, both the current and voltage sensing circuitry are based on integrated circuits (IC) amplifiers. Since the current transducer used for current sensing already produces a small output voltage, the amplifier used for the current sensing circuitry is the well-established and low-cost LM741 [38] in a unity gain configuration [39] for use as a pre-ADC buffer. The voltage sensing circuitry is based on the more specific AD629 differential amplifier [40] since the welder's output voltage cannot be assumed to be referenced to the ground on the current-voltage sensor measurement system. This differential amplifier is another inexpensive IC with a default gain of one and high common-mode voltage limits. This allows the welder ground to float well above the ground of the currentvoltage sensor measurement system without damaging the IC. The rest of the system simply comprises Zener diodes for protection [39], voltage dividers, and smoothing/decoupling capacitors [41].

As can be seen in Figures 1 and 2, the circuitry to measure the welder's voltage and current is straightforward and fits on a circuit board area of approximately 8 sq. as shown in Figure 3.

As shown in Figure 3, all components used are through hole; thus, if miniaturization is desired a new board can be designed with surface mount components to reduce the total board area. Three mounting holes (for size 4 screws) have been placed on the board; however, these were not used during testing since the board was held securely due to the welding cable running through the current transducer and 

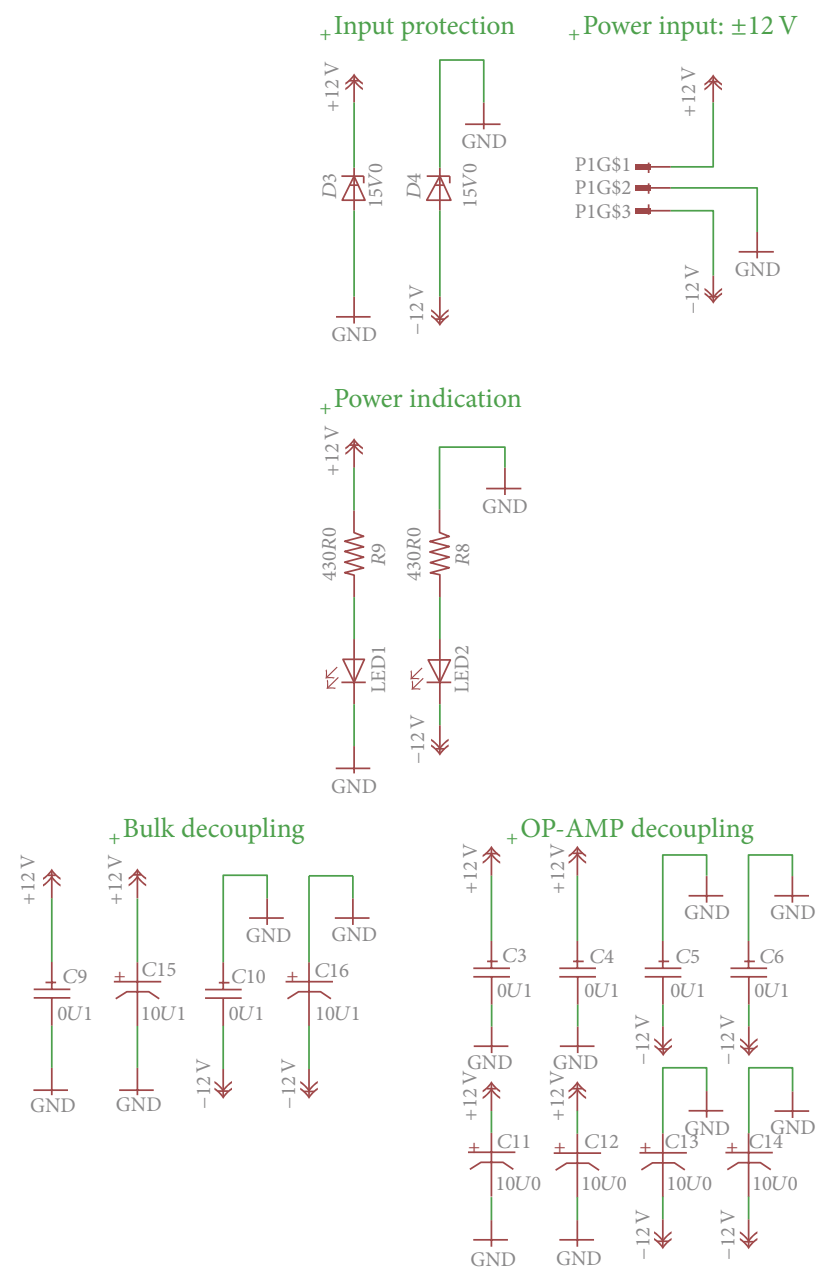

FIGURE 2: Input power protection, indication, and conditioning.

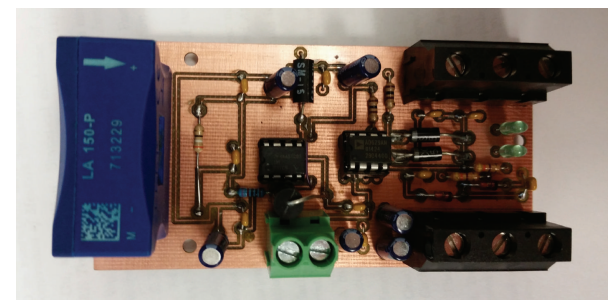

FIGURE 3: Completed circuit.

the positioning of the board in the welder chassis. It should be noted that if these holes are used, nylon (or any other nonconductive material) washers should be used to prevent shorting any circuitry. These holes can be seen on the circuit board artwork given in Appendix D.

The board requires a $\pm 12 \mathrm{~V}$ supply for operation, which is attached to the circuitry through a 3-position $(+12 \mathrm{~V}$, ground, $-12 \mathrm{~V}$ ) screw terminal on the board. These terminals are labeled on the board itself for reference. The board is protected using $15 \mathrm{~V}$ Zener diodes on both rails in an attempt to make the board more robust to any large transients or user error. LED indicators are also present for visual verification that the voltage has been applied to the board.

The current sensing circuitry relies on the use of an offthe-shelf current transducer, which outputs a small current proportional to the relatively large current running through its center; the welder cable inside the chassis was routed through the current transducer's center. A $15 \Omega$ resistor shunts this current to ground, creating a voltage, which can be directly measured. This voltage is buffered with a unity gain op-amp and is routed to an analog input on the Arduino via a screw terminal connector on the board as shown in Figure 3.

The welder voltage is connected to the board via a twoposition screw terminal as shown in Figure 3. The voltage sensing circuitry's first stage is a simple voltage divider, reducing the welder's relatively high output voltage down to a range the electronics and Arduino can tolerate. The scaled voltage from the voltage divider is buffered with a differential amplifier, from which it is routed to an analog input on the Arduino via a screw terminal connector on the board. Note that outputs of both the current sensing circuitry and voltage sensing circuitry are both clamped at $5.1 \mathrm{~V}$ using a Zener diode so that Arduino's inputs are protected from large transients from the welder or sensing circuitry. Both signals are also smoothed with capacitors to eliminate high frequency noise.

It should be noted that there are two separate ground terminals on the circuit board. During testing, it was found that connecting the ground of the Arduino to the second ground terminal on the board caused a ground loop and erratic behavior; thus, it is advised that only the ground from the $\pm 12 \mathrm{~V}$ power supply is connected to the board.

2.2. Testing. The source code runs on an Arduino Uno, which was used as the data acquisition device, which is shown in Appendix B.

The power measurement circuit was installed in the Millermatic 140 welder for this initial test. G-code producing a simple zig-zag weld bead with five-second pauses to permit adjustment of the manual welder voltage setting was written for these tests ( $\mathrm{g}$-code is provided in Appendix C). Aluminum wire, alloy $4043,0.035$ inches $(0.899 \mathrm{~mm})$ in diameter from Blue Demon (heat 34-2879, lot 43909), was used for the aluminum tests. Steel wire from Forney, ER70S-6 mild steel, 0.024 inches $(0.61 \mathrm{~mm})$ in diameter from heat number Y12060609, was used for the steel test. Mild steel 1/4-inch $(6.35 \mathrm{~mm})$ thick substrates that had been used previously for other tests were cleaned and reused for these tests. Wire speed for all the tests was set at position 70 on the welder's manual wire speed adjustment for all tests. The distance between the substrate and the tip of the welding gun nozzle was fixed at $8 \mathrm{~mm}$.

The location of the sensors and $3 \mathrm{D}$ printing welder is shown in Figure 4.

One test was performed at a welding velocity of $70 \mathrm{~mm} / \mathrm{s}$ without shield gas. Two more tests were performed with shield/cover gas, one at a velocity of $70 \mathrm{~mm} / \mathrm{s}$ and one at $90 \mathrm{~mm} / \mathrm{s}$. The "instantaneous" voltage and current reported are the average of 250 individual samples collected once every 


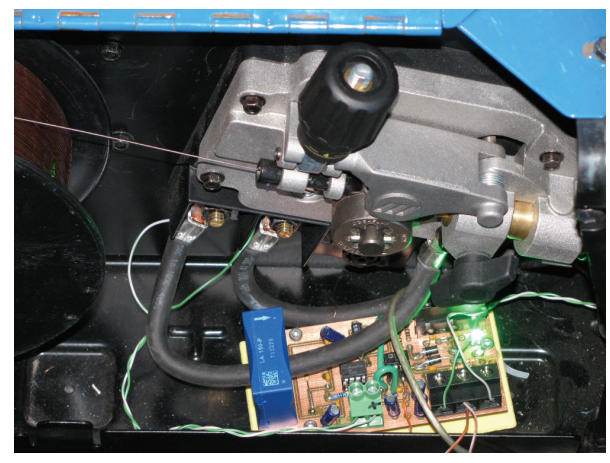

Figure 4: Circuit installed in welder.

few milliseconds, determined by the analog sample time of the microcontroller, or approximately $60 \mathrm{~ms}$.

The program performs ADC measurements in a loop. Because the execution path does not branch and the timing of every ADC measurement is equal, the sample number is directly proportional to the time. However, computing the exact time between samples is complex and it changes with every change to the code. It is therefore better to include a timestamp with every measurement, which will be incorporated in future versions of the firmware.

Welder voltage was initially set at a level of two and was increased in single unit steps during each pause to a maximum level indicated by the arc becoming unstable. This was done to minimize the risk of damaging the welding gun.

\section{Results and Discussion}

The instantaneous voltage and current for the entire duration of each of the three tests are shown in Figures 5, 6, and 7, respectively. Each plateau in the plots indicates a different voltage setting on the welder. At low voltage settings, significant noise is apparent, likely indicating that the welding velocity was too high for those settings, making it impossible to maintain a stable arc. In many cases, the voltage and current decay at the end of a bead segment rather than going directly to zero. This is likely due to the condition of the wire when the welder was shut off at the end of a segment. If the wire was in contact with the substrate, energy rapidly dissipated through a short circuit. If the wire was not in contact with the substrate, energy dissipated more slowly through the welder's internal circuitry.

Composite average voltage and current for each voltage setting and all tests are shown in Figure 8. In all cases, the relationship between measured values and the voltage setting are approximately linear except at the higher voltage settings, when the slope starts to decrease. A comparison of the lines from the test with no gas, which could be conducted at higher voltage settings, to those with cover gas demonstrates that this change in slope is either a product of the process or a control strategy.

The average voltage is plotted against average current and is shown in Figure 9. Figure 9 demonstrates that the relationship is linear as expected, further confirming proper

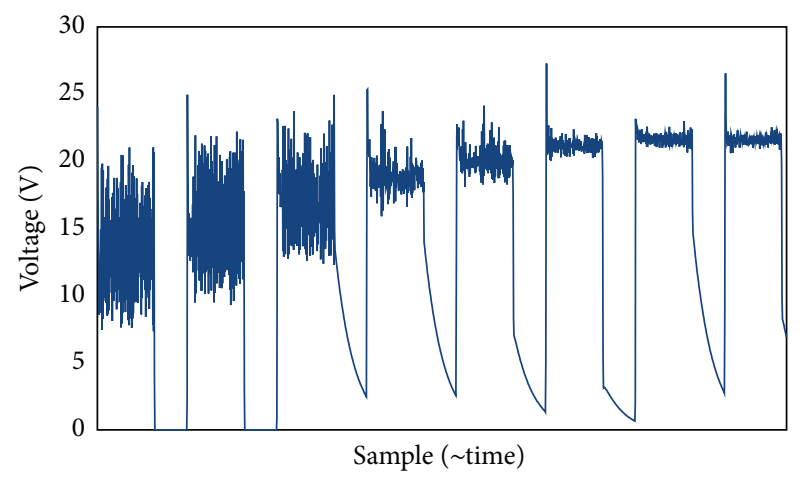

(a)

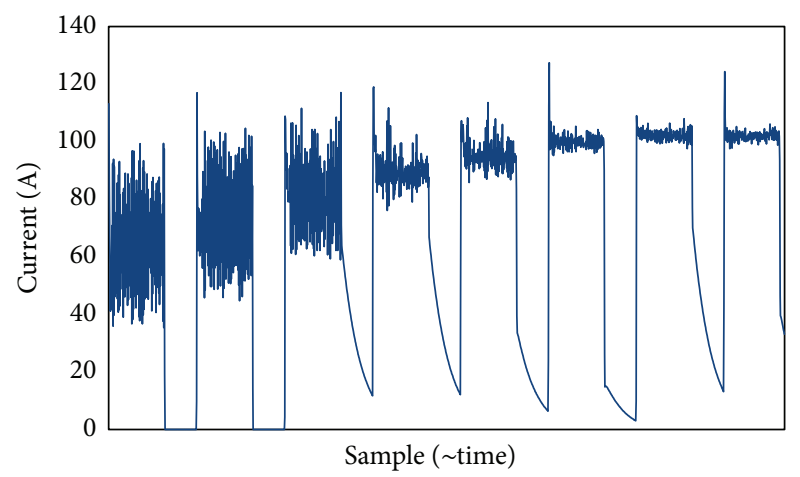

(b)

FIGURE 5: Instantaneous voltage (a) and current (b) measured while welding with steel wire without shield gas at a welding velocity of $70 \mathrm{~mm} / \mathrm{s}$.

operation of the power monitoring circuit. Welder voltage and current were found to be directly proportional to the welder voltage setting until high currents are reached, at which point the slope decreases. This is most likely due to the limitations of the welder at high currents as shown in Figure 8.

Finally, the GMAW 3D printing welder power monitoring circuit and software were refined to capture the instantaneous current and the voltage at a high welding current as a function of time as shown in Figure 10.

The GMAW 3D printing welder power monitoring circuit performed as designed and represents significant savings from even the lowest cost commercial alternative. Commercial alternatives to the power monitoring circuit range in price from $\$ 3,500$ to $\$ 7,000$. These commercial versions offer higher sampling frequencies, up to $10 \mathrm{kHz}$, and custom analysis software. The open-source sensor circuit discussed in this paper costs $\$ 89$ in components, including the milled printed circuit board, and required at most two hours to assemble. This results in savings of a factor of 39 to almost 70 if only the parts are considered and roughly a factor of at least 12 even if $\$ 100 /$ hour labor for the assembly is included in the cost estimation. Such savings would be expected from free and open-source hardware developed for a scientific and engineering application [11]. This creates significant value [42] through the lateral scaling and replication of the sensor device. Finally, it should also be pointed out that these cost 


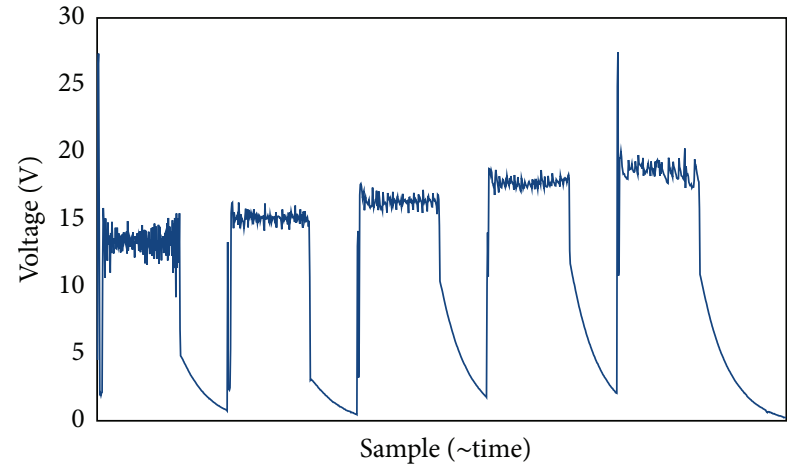

(a)

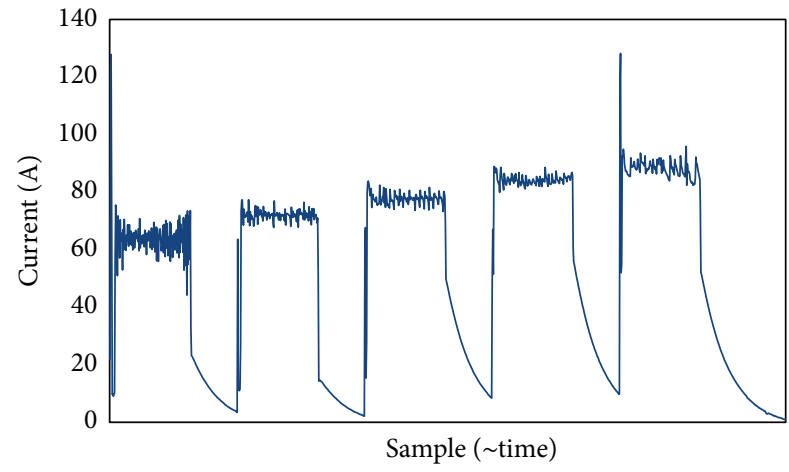

(b)

FIGURE 6: Instantaneous voltage (a) and current (b) measured while welding with aluminum wire and $100 \%$ argon shield gas at a welding velocity of $70 \mathrm{~mm} / \mathrm{s}$.

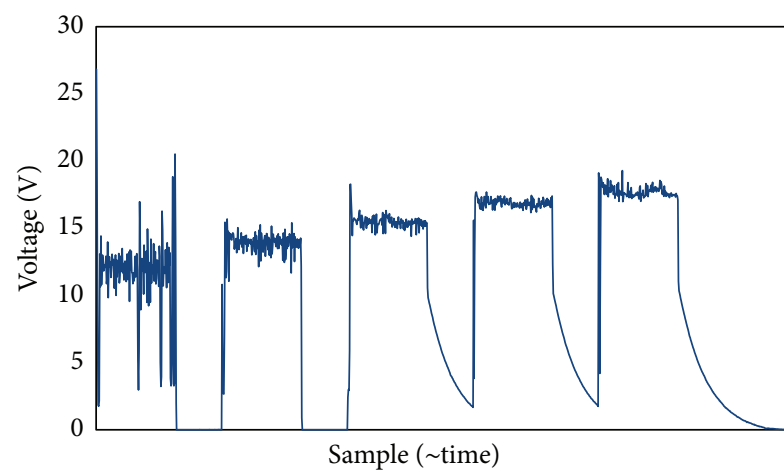

(a)

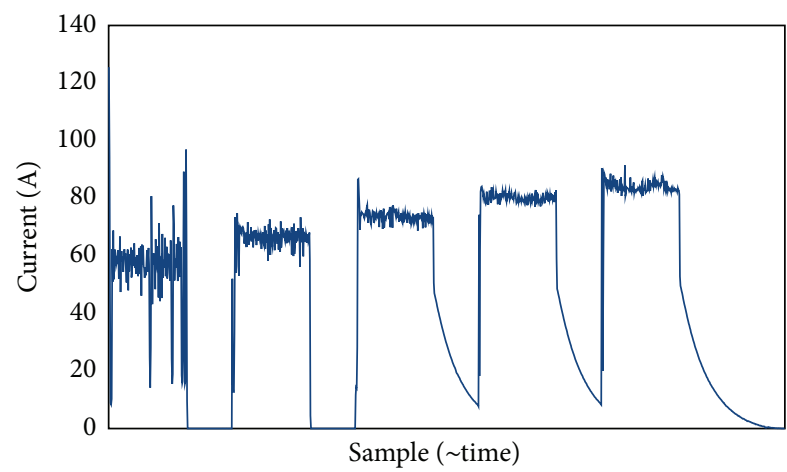

(b)

Figure 7: Instantaneous voltage (a) and current (b) measured while welding with aluminum wire and $100 \%$ argon shield gas at a welding velocity of $90 \mathrm{~mm} / \mathrm{s}$.

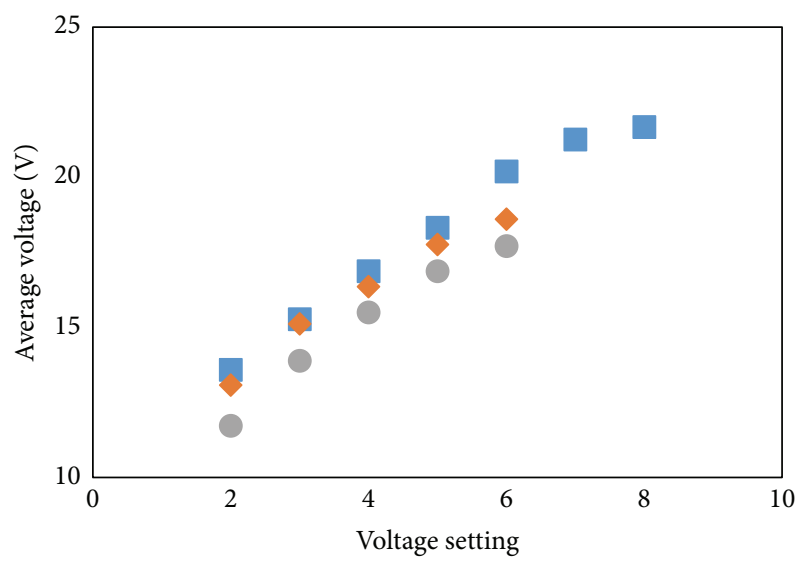

Steel-steel, 70, no gas

Al-steel, 70, Ar gas

- Al-steel, 90, Ar gas

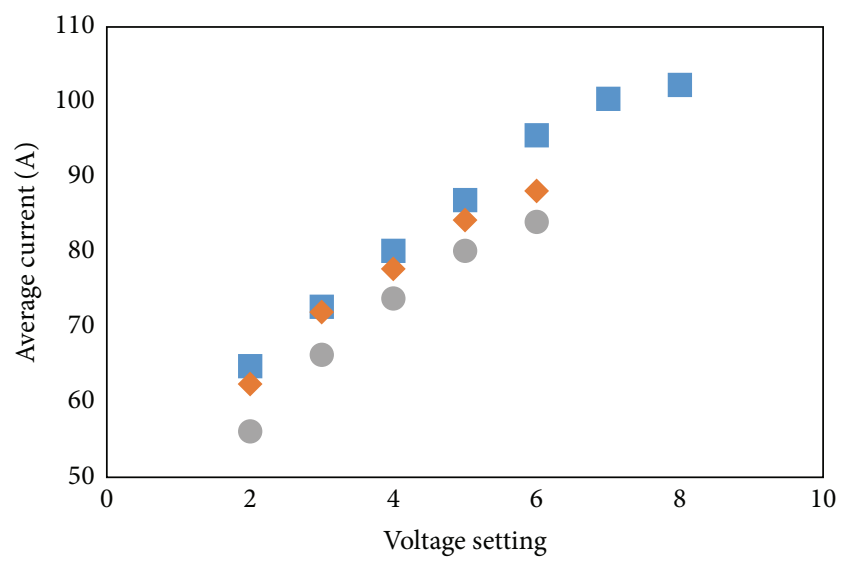

Steel-steel, 70, no gas

Al-steel, 70, Ar gas

Al-steel, 90, Ar gas

FIGURE 8: Average voltage (a) and current (b) as a function of voltage setting for each of the tests performed. 


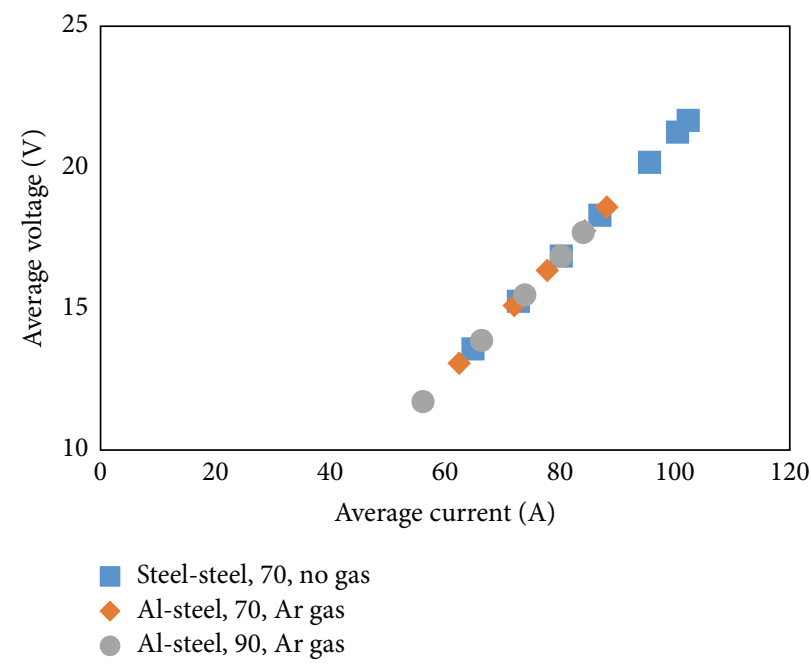

FIGURE 9: Average voltage versus average current for the three tests.

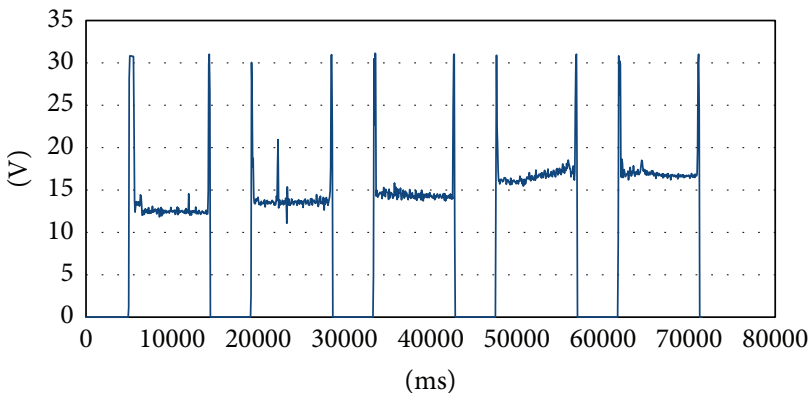

(a)

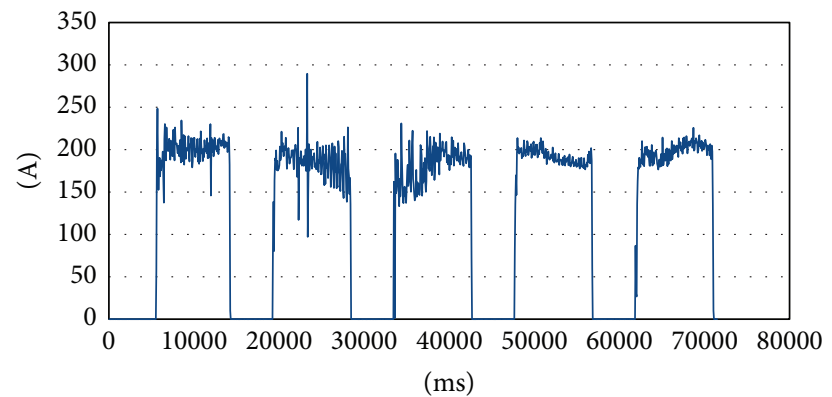

(b)

FIGURE 10: Instantaneous voltage (a) and current (b) measured as a function of time with a high current setting.

estimates are conservative as Arduino clones are available for as little as $\$ 3.00$ and if two analog channels are converted a sample frequency of $4 \mathrm{kHz}$ is possible. If it is later found to be important for this application, using one Arduino for each analog channel increases sample frequency to $16 \mathrm{kHz}$ to outperform off-the-solutions for GMAW monitoring. The design could be used by any processing platform that has the ability to read two analog channels in the range of $0-5 \mathrm{~V}$.

Although the open-source sensor platform performed adequately for its intended purpose, the design offers several opportunities for future improvement. First, the board itself can be fabricated with a new functionality of RepRap systems that include PCB milling. Secondly, the data acquisition code lacked a time stamp making it hard to display a time scale with the samples. Future work with the monitor should include a time stamp with every measurement. The Arduino code could be moved into 3D printer control firmware, so it can use the measurements in a closed loop system to get better print quality, for example, arc length control. Future improvements on the current-voltage sensor measurement system include an electrical isolator to completely isolate the Arduino from the relatively noisy welding circuitry and ferrite clamps on long cable runs to attenuate electromagnetic interference from the welder arcs. These upgrades will make the circuitry more robust to noise and any ground bounce or ground loops within the circuitry.

\section{Conclusions}

The welder power sensor monitoring circuit performed as designed and enables in situ current, voltage, and power monitoring of a GMAW 3D printer. Welder voltage and current were found to be directly proportional to the welder voltage setting until high currents are reached, at which point the slope decreases. There is no significant difference in voltage setting versus power output when comparing a steel electrode to an aluminum electrode and welding velocity or whether shield gas is used or not. It is therefore possible to interpolate approximate voltage and current from a given setting without direct measurement. The open-source sensor circuit costs $\$ 89$ in components, including the milled printed circuit board, and required at most two hours to assemble. This results in substantial savings compared to proprietary equipment. This creates significant potential value through the lateral scaling and replication of the sensor device in the $3 \mathrm{D}$ printing community. Since the hardware and software are open-source, both can be modified by end users to maximize their value for that user's particular application. Likewise, 
capabilities to match or surpass any similar sensor system for GMAW can be added as needed with relative ease.

\section{Disclosure}

The US Government is authorized to reproduce and distribute reprints for Governmental purposes notwithstanding any copyright notation thereon.

\section{Disclaimer}

The views and conclusions contained herein are those of the authors and should not be interpreted as necessarily representing the official policies or endorsements, either expressed or implied, of Air Force Research Laboratory or the US Government.

\section{Conflict of Interests}

The authors declare that there is no conflict of interests regarding the publication of this paper.

\section{Acknowledgments}

The authors would like to acknowledge helpful discussions with C. Hsu at Miller Electric and America Makes team members. The authors would also like to acknowledge support and technical assistance from the Miller Electric Manufacturing Company. This material is based on research sponsored by Air Force Research Laboratory under Agreement no. FA8650-12-2-7230.

\section{References}

[1] R. Jones, P. Haufe, E. Sells et al., "Reprap-the replicating rapid prototyper," Robotica, vol. 29, no. 1, pp. 177-191, 2011.

[2] E. Sells, Z. Smith, S. Bailard, A. Bowyer, and V. Olliver, "RepRap: the replicating rapid prototyper: maximizing customizability by breeding the means of production," in Handbook of Research in Mass Customization and Personalization, World Scientific Publishing, 2009.

[3] C. Baechler, M. DeVuono, and J. M. Pearce, "Distributed recycling of waste polymer into RepRap feedstock," Rapid Prototyping Journal, vol. 19, no. 2, pp. 118-125, 2013.

[4] E. Hunt, C. Zhang, N. Anzalone, and J. M. Pearce, "Polymer recycling codes for distributed manufacturing with 3-D printers," Resources, Conservation and Recycling, vol. 97, pp. 24-30, 2015.

[5] G. C. Anzalone, C. Zhang, B. Wijnen, P. G. Sanders, and J. M. Pearce, "A low-cost open-source metal 3-D printer," IEEE Access, vol. 1, pp. 803-810, 2013.

[6] A. Haselhuhn, E. Gooding, A. Glover et al., "Substrate release mechanisms for gas metal arc weld 3D aluminum metal printing," 3D Printing and Additive Manufacturing, vol. 1, no. 4, pp. 204-209, 2014.

[7] T. Baden, A. M. Chagas, G. Gage, T. Marzullo, L. L. PrietoGodino, and T. Euler, "Open labware: 3-D printing your own lab equipment," PLoS Biology, vol. 13, no. 3, 2015.

[8] J. M. Pearce, "Building research equipment with free, opensource hardware," Science, vol. 337, no. 6100, pp. 1303-1304, 2012.
[9] D. K. Fisher and P. J. Gould, "Open-source hardware is a lowcost alternative for scientific instrumentation and research," Modern Instrumentation, vol. 1, no. 2, pp. 8-20, 2012.

[10] C. Zhang, N. C. Anzalone, R. P. Faria, and J. M. Pearce, "Opensource 3D-printable optics equipment," PLoS ONE, vol. 8, no. 3, Article ID e59840, 2013.

[11] J. M. Pearce, Open-Source Lab: How to Build Your Own Hardware and Reduce Research Costs, Elsevier, New York, NY, USA, 2013.

[12] A. D’Ausilio, "Arduino: a low-cost multipurpose lab equipment," Behavior Research Methods, vol. 44, no. 2, pp. 305-313, 2012.

[13] J. Busquets, J. V. Busquets, D. Tudela et al., "Low-cost AUV based on Arduino open source microcontroller board for oceanographic research applications in a collaborative long term deployment missions and suitable for combining with an USV as autonomous automatic recharging platform," in Proceedings of the IEEE/OES Autonomous Underwater Vehicles (AUV '12), pp. 1-10, IEEE, 2012.

[14] S.-H. Chiu and P. L. Urban, "Robotics-assisted mass spectrometry assay platform enabled by open-source electronics," Biosensors and Bioelectronics, vol. 64, pp. 260-268, 2015.

[15] J.-B. Hu, T.-R. Chen, C.-H. Chang, J.-Y. Cheng, Y.-C. Chen, and P. L. Urban, "A compact 3D-printed interface for coupling open digital microchips with Venturi easy ambient sonic-spray ionization mass spectrometry," Analyst, vol. 140, no. 5, pp. 14951501, 2015.

[16] E. J. Gualda, T. Vale, P. Almada, J. A. Feijó, G. G. Martins, and N. Moreno, "OpenSpinMicroscopy: an open-source integrated microscopy platform," Nature Methods, vol. 10, no. 7, pp. 599600, 2013.

[17] P. Teikari, R. P. Najjar, H. Malkki et al., "An inexpensive Arduino-based LED stimulator system for vision research," Journal of Neuroscience Methods, vol. 211, no. 2, pp. 227-236, 2012.

[18] O. Pineño, "ArduiPod Box: a low-cost and open-source Skinner box using an iPod Touch and an Arduino microcontroller," Behavior Research Methods, vol. 46, no. 1, pp. 196-205, 2014.

[19] J. Kentzer, B. Koch, M. Thiim, R. W. Jones, and E. Villumsen, "An open source hardware-based mechatronics project: the replicating rapid 3-D printer," in Proceedings of the 4th International Conference on Mechatronics (ICOM '11), pp. 1-8, IEEE, 2011.

[20] M. Gopalakrishnan and M. Gühr, "A low-cost mirror mount control system for optics setups," American Journal of Physics, vol. 83, no. 2, pp. 186-190, 2015.

[21] J. Sáiz, I. J. Koenka, C. García-Ruiz, B. Müller, T. Chwalek, and P. C. Hauser, "Micro-injector for capillary electrophoresis," Electrophoresis, 2015.

[22] P. Nikolaou, A. M. Coffey, L. L. Walkup et al., "Near-unity nuclear polarization with an open-source 129Xe hyperpolarizer for NMR and MRI," Proceedings of the National Academy of Sciences of the United States of America, vol. 110, no. 35, pp. 14150-14155, 2013.

[23] M. P. Walzik, V. Vollmar, T. Lachnit et al., "A portable low-cost long-term live-cell imaging platform for biomedical research and education," Biosensors and Bioelectronics, vol. 64, pp. 639649, 2015.

[24] S. Trilles, A. Luján, Ó. Belmonte, R. Montoliu, J. TorresSospedra, and J. Huerta, "SEnviro: a sensorized platform proposal using open hardware and open standards," Sensors, vol. 15, no. 3, pp. 5555-5582, 2015.

[25] G. C. Anzalone, A. G. Glover, and J. M. Pearce, "Open-source colorimeter," Sensors, vol. 13, no. 4, pp. 5338-5346, 2013. 
[26] B. Wijnen, G. C. Anzalone, and J. M. Pearce, "Open-source mobile water quality testing platform," Journal of Water, Sanitation and Hygiene for Development, vol. 4, no. 3, pp. 532-537, 2014.

[27] L. Russell, A. L. Steele, and R. Goubran, "Low-cost, rapid prototyping of IMU and pressure monitoring system using an open source hardware design," in Proceedings of the IEEE International Instrumentation and Measurement Technology Conference (I2MTC '12), pp. 2695-2699, IEEE, Graz, Austria, May 2012.

[28] A. H. Shajahan and A. Anand, "Data acquisition and control using arduino-android platform: smart plug," in Proceedings of the International Conference on Energy Efficient Technologies for Sustainability (ICEETS '13), pp. 241-244, April 2013.

[29] R. Sun, M. B. Bouchard, and E. M. C. Hillman, "SPLASSH: open source software for camera-based high-speed, multispectral invivo optical image acquisition," Biomedical Optics Express, vol. 1, no. 2, pp. 385-397, 2010.

[30] R. S. Ferrarezi, S. K. Dove, and M. W. van Iersel, "An automated system for monitoring soil moisture and controlling irrigation using low-cost open-source microcontrollers," HortTechnology, vol. 25, no. 1, pp. 110-118, 2015.

[31] Santoshkumar, K. Chelli, and S. Chavhan, "Development of wireless sensor node to monitor poultry farm," in Mobile Communication and Power Engineering, vol. 296 of Communications in Computer and Information Science, pp. 27-32, Springer, Berlin, Germany, 2013.

[32] M. Hirafuji, H. Yoichi, T. Kiura et al., "Creating highperformance/low-cost ambient sensor cloud system using OpenFS (open field server) for high-throughput phenotyping," in Proceedings of the SICE Annual Conference (SICE '11), pp. 2090-2092, IEEE, 2011.

[33] I. J. Koenka, J. Sáiz, and P. C. Hauser, "Instrumentino: an opensource modular Python framework for controlling Arduino based experimental instruments," Computer Physics Communications, vol. 185, no. 10, pp. 2724-2729, 2014.

[34] H. Lim, J. Park, D. Lee, and H. J. Kim, "Build your own quadrotor: open-source projects on unmanned aerial vehicles," IEEE Robotics \& Automation Magazine, vol. 19, no. 3, pp. 33-45, 2012.

[35] J. Gonzalez-Gomez, A. Valero-Gomez, A. Prieto-Moreno, and M. Abderrahim, "A new open source 3d-printable mobile robotic platform for education," in Advances in Autonomous Mini Robots, pp. 49-62, Springer, Berlin, Germany, 2012.

[36] J.-D. Warren, J. Adams, and H. Molle, "Arduino for robotics," in Arduino Robotics, pp. 51-82, Apress, 2011.

[37] R. Fobel, C. Fobel, and A. R. Wheeler, "DropBot: an opensource digital microfluidic control system with precise control of electrostatic driving force and instantaneous drop velocity measurement," Applied Physics Letters, vol. 102, no. 19, Article ID 193513, 2013.

[38] Texas Instruments, LM741 Operational Amplifier, LM741 Datasheet, 1998.

[39] A. Sedra and K. Smith, Microelectronic Circuits, Oxford University Press, New York, NY, USA, 2010.

[40] Analog Devices, "High common-mode voltage, difference amplifier," AD629 datasheet, Rev. C, 2011.

[41] J. Irwin and R. Nelms, Basic Engineering Circuit Analysis, John Wiley \& Sons, Hoboken, NJ, USA, 2005.

[42] J. M. Pearce, "Quantifying the value of open source hardware development," Modern Economy, vol. 6, no. 1, pp. 1-11, 2015. 

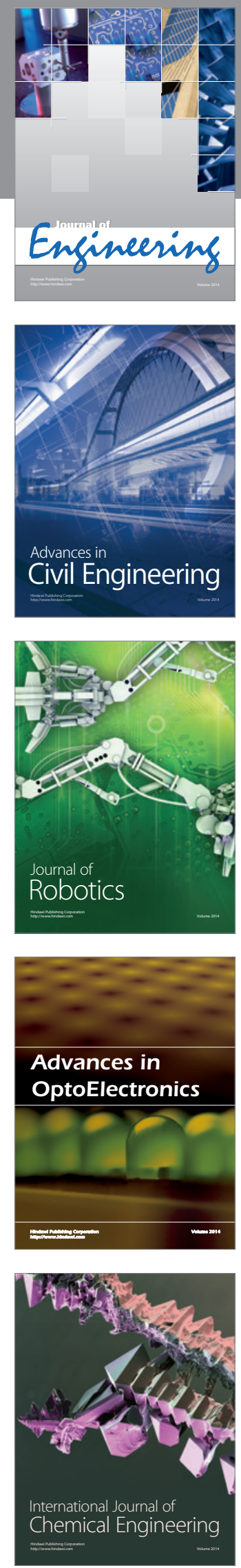

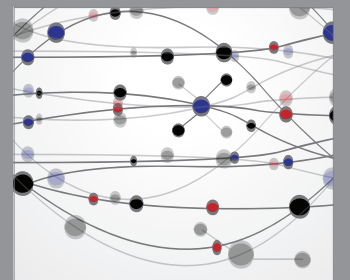

The Scientific World Journal
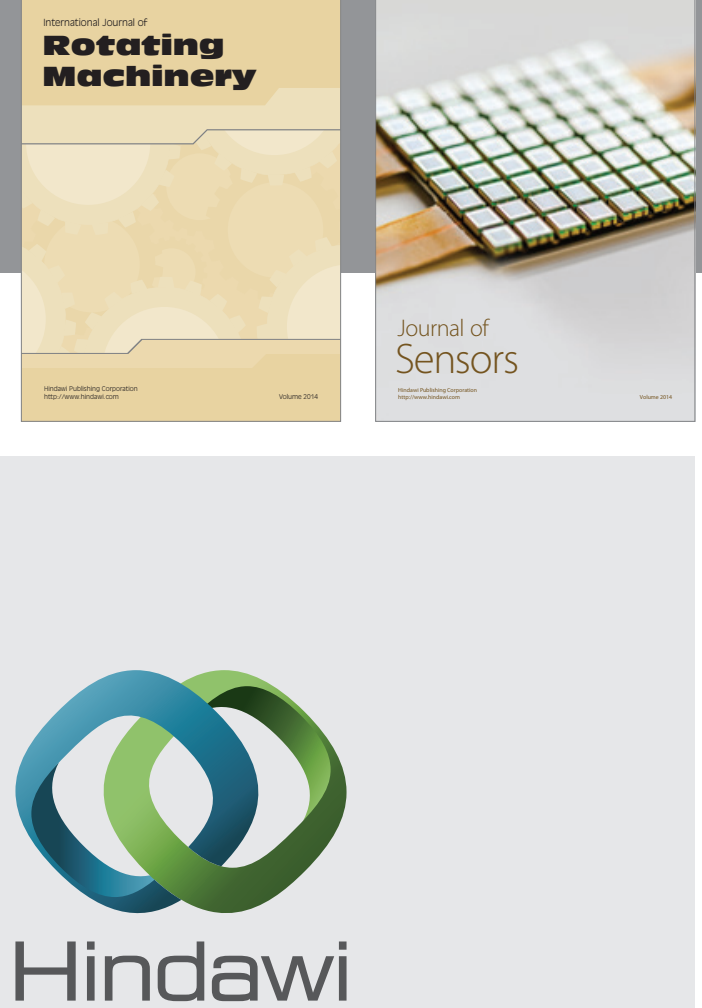

Submit your manuscripts at http://www.hindawi.com
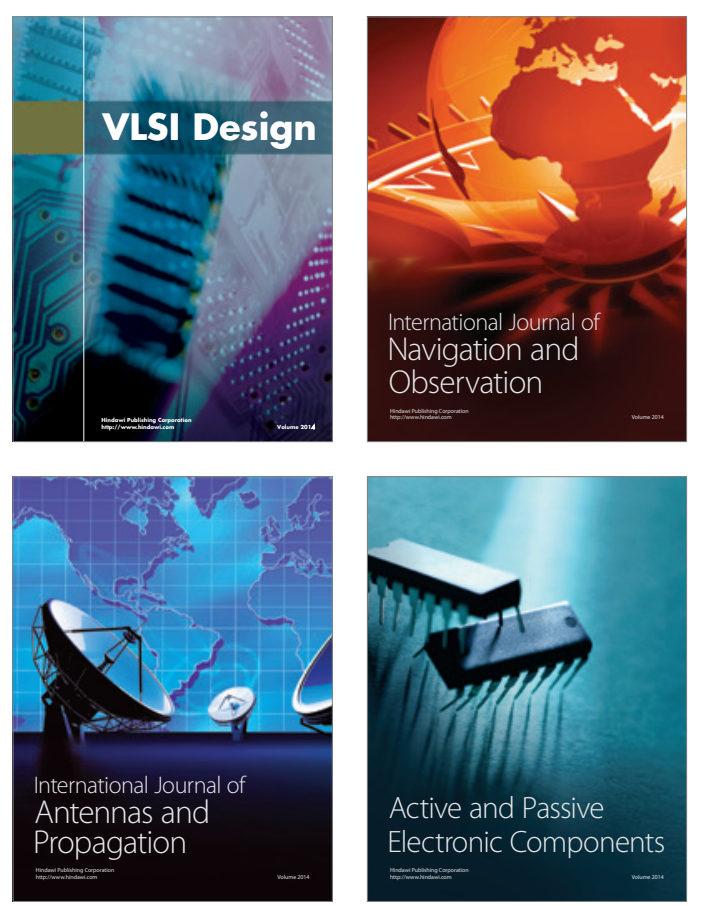
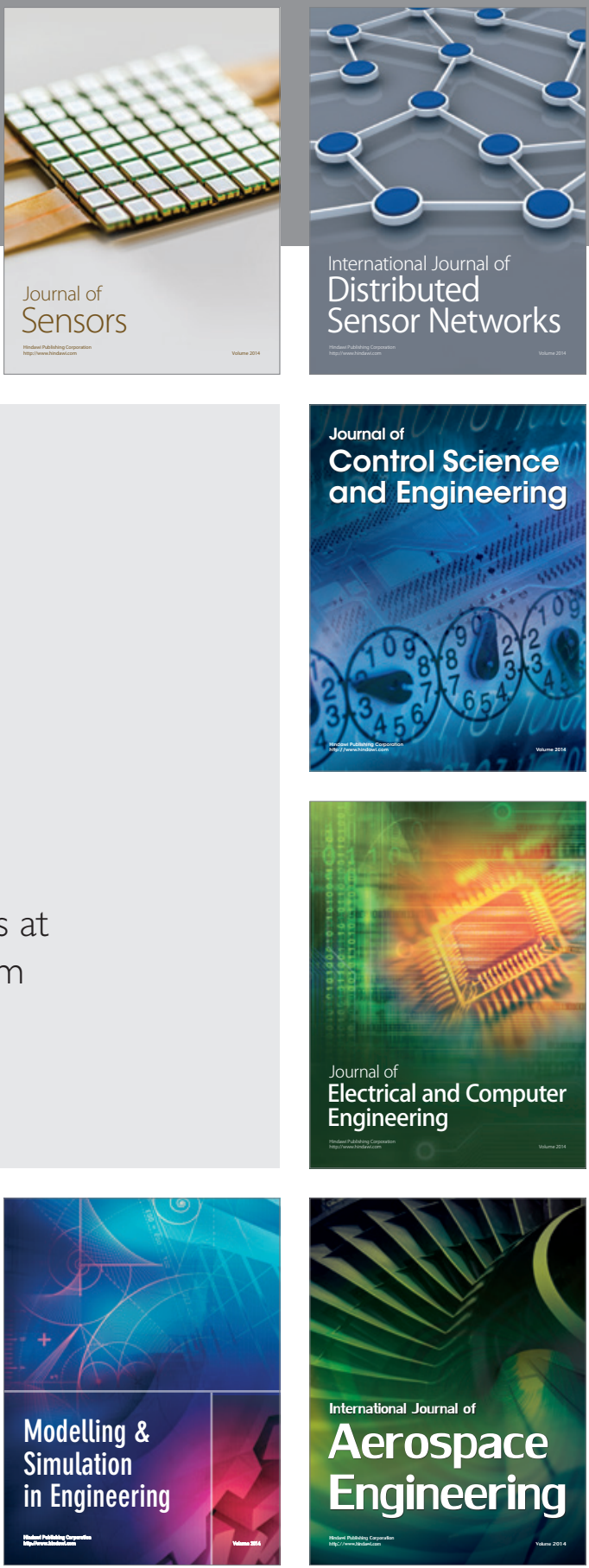

Journal of

Control Science

and Engineering
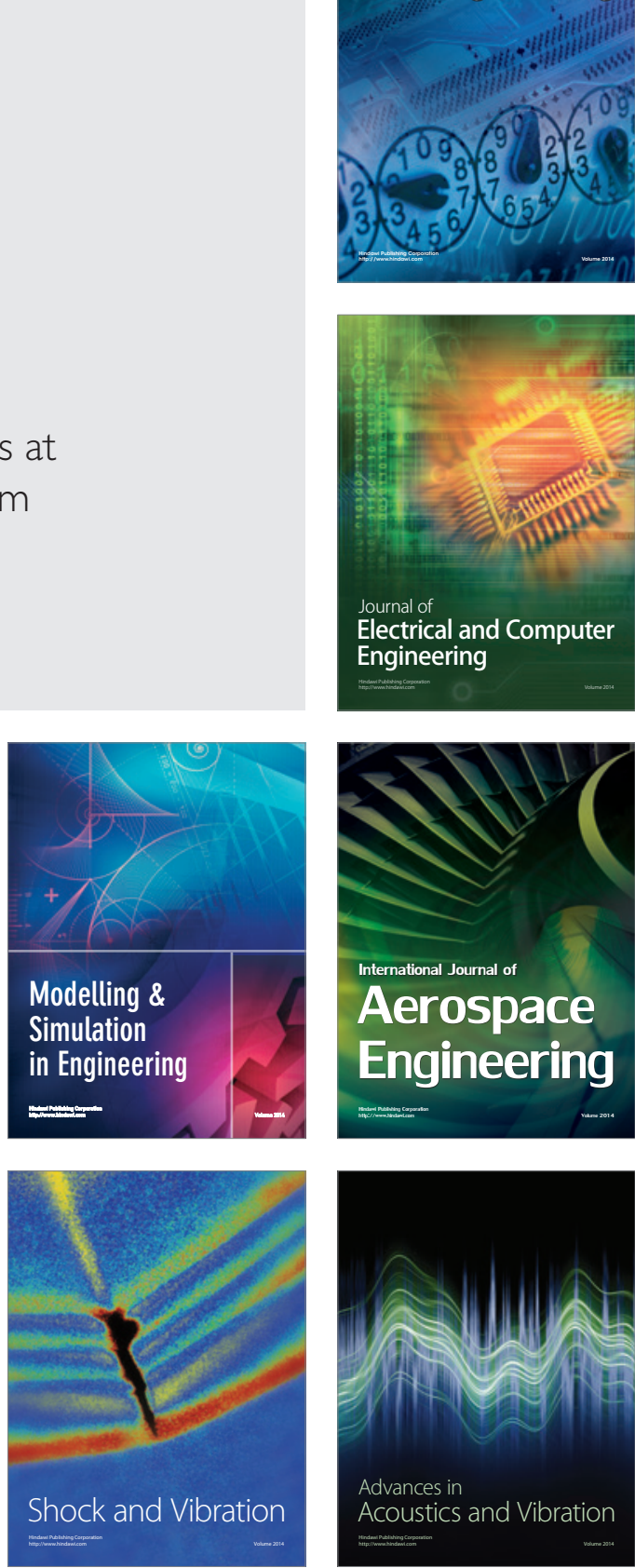